\title{
Surgical Management and Long-Term Follow-Up of a Giant Hepatic Cyst with an Internal Septum in a
}

\section{Cat}

\author{
Kihoon $\mathrm{Kim}^{1,2}$ \\ Hyungjoon $\mathrm{Kim}^{2}$ \\ Ki-Dong Eom ${ }^{3}$ \\ Hwi-Yool Kim ${ }^{1, *}$ \\ 'Department of Veterinary Surgery, \\ College of Veterinary Medicine, Konkuk \\ University, Seoul 05029, Korea \\ ${ }^{2}$ Department of Surgery, Baeksan Feline \\ Medical Center, Seoul 06223, Korea \\ ${ }^{3}$ Department of Veterinary Radiology and \\ Diagnostic Imaging, College of Veterinary \\ Medicine, Konkuk University, Seoul 05029, \\ Korea
}

Abstract A 1-year-old spayed female Scottish Fold cat presented with a 1.5-month history of vomiting, intermittent dyspnea, and abdominal distention. Radiographic, ultrasonographic, and computed tomographic examinations suggested a tentative diagnosis of a fluid-containing cystic mass with an internal septum. The mass was surgically removed. Histological examinations revealed that the mass was a non-neoplastic hepatic cyst. The patient had no recurrence of the cystic structure but died of acute renal failure 5 years after the surgery. This report describes the unusual case of a giant hepatic cyst with clinical signs that resolved after mass removal.

Key words hepatic, cyst, septum, cat.
*Correspondence: hykim@konkuk.ac.kr

\section{ORCID}

Kihoon Kim:

https://orcid.org/0000-0003-2529-7878 Hyungjoon Kim:

https://orcid.org/0000-0002-3059-8969

Ki-Dong Eom:

https://orcid.org/0000-0001-5032-5311

Hwi-Yool Kim:

https://orcid.org/0000-0001-6237-9958

Copyright $\odot$ The Korean Society of Veterinary Clinics
Received January 25, 2021 / Accepted November 29, 2021

This is an open access article distributed under the terms of the Creative Commons Attribution Non-Commercial License (http://creativecommons.org/licenses/ by-nc/4.0) which permits unrestricted non-commercial use, distribution, and reproduction in any medium, provided the original work is properly cited. 


\section{Introduction}

Hepatic cysts are the most frequently occurring, benign, space-occupying malformations. However, if rapidly growing hepatic cysts contain a septal structure, they are difficult to correctly distinguish from malignant cystadenocarcinomas (9). A previous human study reported a case of biliary cystadenocarcinoma followed up as a simple hepatic cyst (1). In cats, hepatic vascular cysts of neoplastic origin, such as hemangiomas or hemangiosarcomas, are common (4). Therefore, in cases of hepatic cysts, an animal's history, clinical signs, imaging findings, as well as laboratory and histopathological data have to be analyzed before arriving at a final diagnosis (6). Giant hepatic cysts generally require treatment if they become large enough to cause clinical symptoms such as jaundice, abdominal pain, nausea, vomiting, and abdominal distention (5).

\section{Case Report}

A 1-year-old spayed female Scottish Fold cat weighing 3.5 $\mathrm{kg}$ was referred to the Konkuk University Veterinary Medical Teaching Hospital for the evaluation of a 1.5-month history of abdominal distention, vomiting, and intermittent dyspnea. The patient was vaccinated, dewormed, and free of heartworm infection. On history taking, the cat's owner reported observing rapid abdominal distention starting 1.5 months previously. A physical examination revealed remarkable abdominal distention. A routine laboratory examination yielded no significant findings. However, radiographic and abdominal ultrasonographic examinations revealed an anechogenic cystic mass that occupied nearly half of the abdomen (Fig. 1A, $B)$. The borders of the mass could not be distinguished from the liver parenchyma, and the mass contained a thin septal structure. The other abdominal organs appeared normal on ultrasonography; however, they had deviated to the caudal part of the abdomen because of compression by the mass. Abdominal computed tomography also revealed the capsule of the hepatic cyst originating from the liver in the transverse view; however, the lobe of the liver attached to the mass could not be identified (Fig. 1C). The cystic wall showed no contrast enhancement. The mass had a maximum length of $14 \mathrm{~cm}$.

On the basis of the radiologic, ultrasonographic, and computed tomographic findings, the cat was tentatively diagnosed with a simple hepatic cyst. However, owing to the rapid progression of abdominal distention and the possibility of development of more severe clinical signs, such as jaundice or cystic rupture, in the near future $(2,8,10)$, the cyst was urgently removed to arrive at a definitive histopathological diagnosis.

The cat received a preoperative intravenous (IV) injection of $0.3 \mathrm{mg} / \mathrm{kg}$ midazolam (Vascam; Hana Pharmaceutical Co. Ltd., Korea) and 0.2 mg/kg butorphanol (Butophan; Myungmoon Pharmaceutical Co. Ltd., Korea). General anesthesia was induced via endotracheal intubation after induction with an IV injection of $6 \mathrm{mg} / \mathrm{kg}$ propofol (Anepol; Hana Pharmaceutical Co. Ltd.). Inhalation anesthesia was maintained
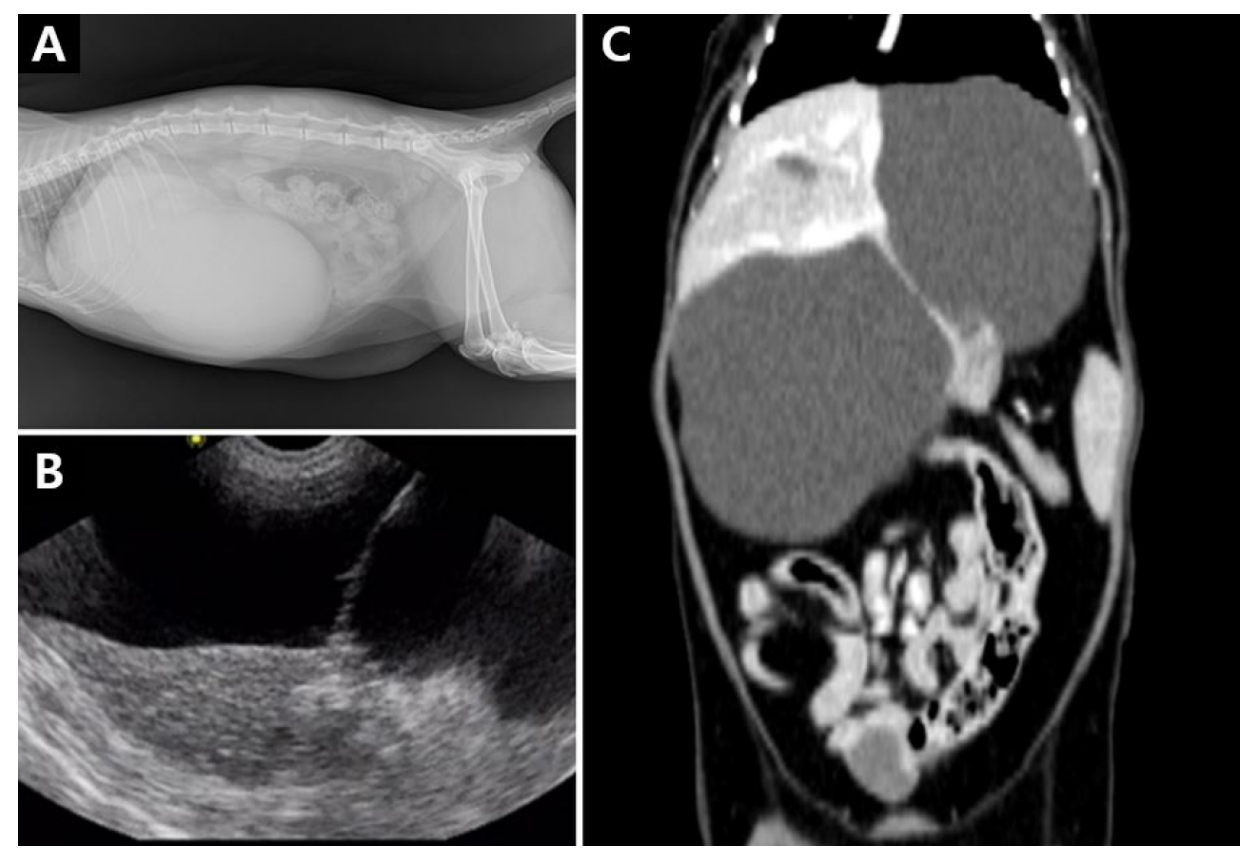

Fig. 1. (A) Lateral abdominal radiography showing a round mass and caudal deviation of the other organs due to compression by the mass. (B) Ultrasonography and (C) preoperative computed tomography reveal the presence of an internal septum within the hepatic cyst. 

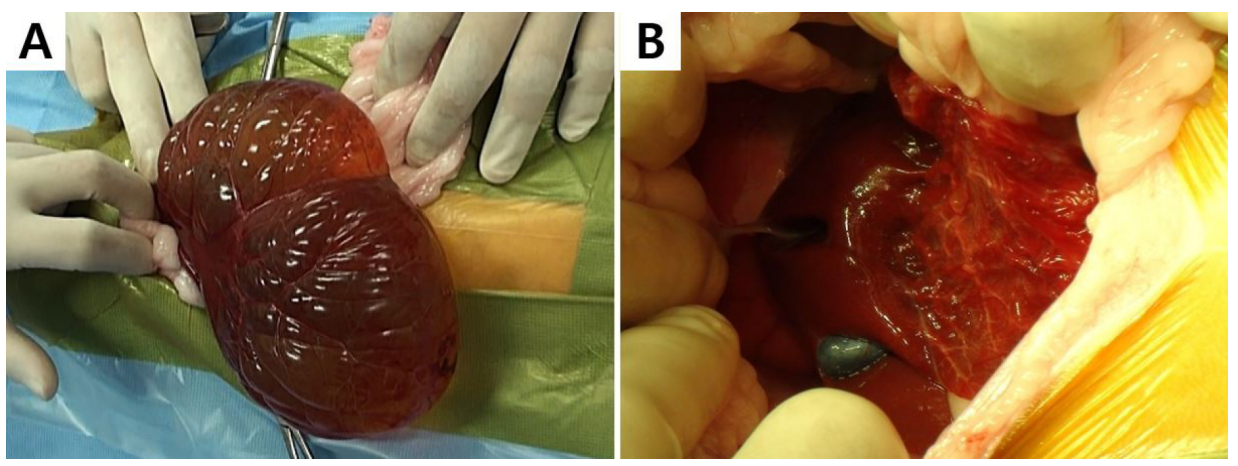

Fig. 2. (A) Gross appearance of hepatic cyst-like structures removed through an abdominal incision during surgery in a 1-yearold cat. (B) Appearance of the cyst-like structures originating from the left lateral liver lobe after fluid removal.
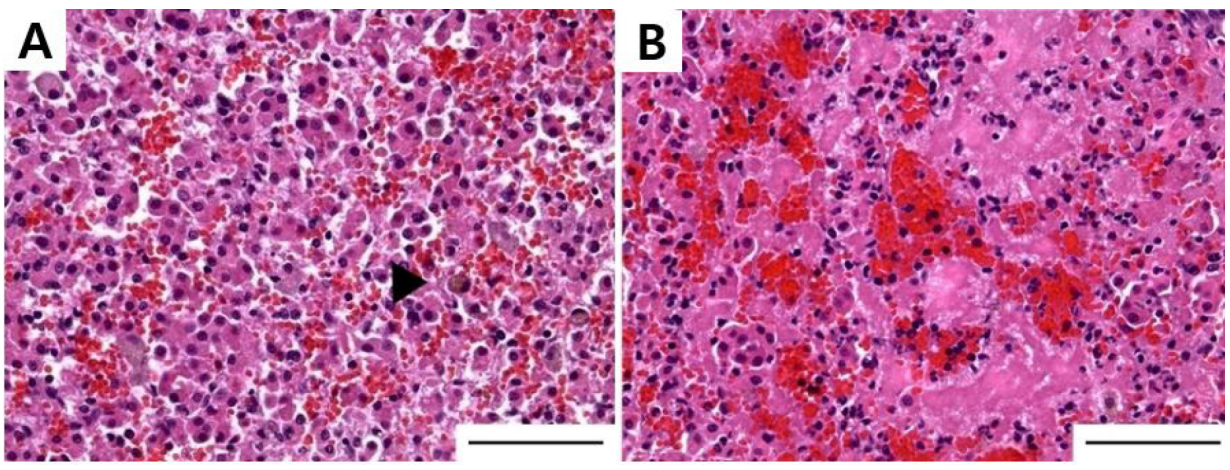

Fig. 3. (A) Hemosiderin-laden hepatocyte (black arrowhead); hematoxylin and eosin $(\mathrm{H} \& \mathrm{E})$ staining $\times 100($ scale bar $=100 \mu \mathrm{m})$. (B) Hemorrhage in the liver parenchyma and loss of normal lobular organization; H\&E staining $\times 100$ (scale bar $=100 \mu \mathrm{m})$.

using a combination of $2 \%$ isoflurane (Forane; ChoongWae Pharmaceutical Co., Korea) and $100 \%$ oxygen. A skin incision was made from the xiphoid to the umbilicus. The cyst was exposed after retracting the omentum (Fig. 2A). The fluid was aspirated using a 24-gauge needle before the surgical removal of the cyst. No communication was noted between the two compartments separated by the internal septum. The cyst originating from the left lateral lobe of the liver was identified (Fig. 2B). Partial lobectomy of the liver, including the cystic structure, was performed. Routine abdominal closure was performed thereafter. As an analgesic, butorphanol $(0.2 \mathrm{mg} / \mathrm{kg}, \mathrm{IV})$ was administered postoperatively every 8 $\mathrm{h}$ for 3 days, and cephalexin $(22 \mathrm{mg} / \mathrm{kg}$ ) was administered twice a day for 10 days.

The fluid within the hepatic cyst was yellow and clear. The bilirubin concentration in the fluid was $0.18 \mathrm{mg} / \mathrm{dL}$, which was four times higher than that in the serum. The remaining fluid parameters were unremarkable when compared to the serum reference range. The total volume of the aspirated fluid was $472.5 \mathrm{~mL}$, which was approximately one-sixth of the cat's body weight.

A liver biopsy sample was acquired from the region adjacent to the cystic structure and was stained with hematoxylin and eosin. Histopathological examination revealed hemorrhage, necrosis of hepatocytes, hemosiderin-laden hepatocytes, and loss of normal lobular organization (Fig.
3A, B). The cystic membrane was also sampled and stained with hematoxylin and eosin. It was 100- to 200- $\mu \mathrm{m}$ thick and lined by a simple continuous layer of squamous cells on the interior surface. A final diagnosis of a simple hepatic cyst with an internal septum was made. Three years after the surgery, the patient remained clinically normal, and no subsequent complications were reported by the owner. According to the referring veterinarian, the patient died 5 years after the surgery because of acute renal failure due to bilateral ureteral obstructions. However, no recurrence of the hepatic cyst was identified throughout the patient's life.

\section{Discussion}

In humans, the incidence of hepatic cysts based on autopsy and ultrasonography is $0.1-0.5 \%$ and $2.5 \%$, respectively (5). However, the incidence of hepatic cysts in cats has not been reported. Hepatic cysts have been categorized as follows: congenital, neoplastic, inflammatory, and traumatic (5). Simple congenital cysts may be solitary $(11)$ or multiple $(6,10)$ and are believed to result from an abnormality in the in utero development of the intrahepatic bile ducts (3). The cyst presented in this case report was solitary and bilocular.

The majority of hepatic cysts present no clinical signs. Therefore, the cysts are generally discovered incidentally or at necropsy unless they become enlarged enough to present 
abdominal distention. However, if clinical signs such as abdominal pain, vomiting, or abdominal distention are present, hepatic cysts should be suspected. A previous human study reported obstructive jaundice resulting from a giant hepatic cyst (3).

For the management of the mass, percutaneous aspiration as the sole treatment before surgery was not considered because this was not a definite therapy (7). Moreover, in biliary cystadenocarcinoma, the possibility of peritoneal seeding by puncture exists (9). In this case, during the surgery, percutaneous aspiration of the fluid was initially attempted using a 24-gauge needle, which tore the cystic membrane. Therefore, complications like mass rupture should be considered before percutaneous aspiration or deroofing during surgery. The high bilirubin concentration in the aspirated fluid was presumed to be a consequence of the compression of the biliary system by the cystic mass. By examining the aspirated fluid, we could also rule out a parasitic cyst or hepatic abscess that resembles a simple hepatic cyst.

Owing to the rapid growth characteristic and presence of an internal septum, it was difficult to determine whether the cyst was a benign simple hepatic cyst or a cavitary neoplasm, such as biliary cystadenoma or cystadenocarcinoma, without performing further histological examination. According to the human medical literature, internal septation of cysts is a typical characteristic of biliary cystadenoma or cystadenocarcinoma (9). A case of biliary cystadenocarcinoma initially diagnosed as a simple hepatic cyst has also been reported in humans (1). The human medical literature also suggests that a simple hepatic cyst is covered by a smooth thin wall rather than a thick fibrous capsule on computed tomography (9). The present case demonstrated the coexistence of the typical characteristics of a simple hepatic cyst and cavitary neoplasm, and the cystic mass had to be removed together with a portion of the left lateral liver lobe, which appeared grossly normal. Complete excision of the left lateral liver lobe was considered unwise until final diagnosis via a histological examination. Additional surgery was not required in this case because histopathological examination of the liver biopsy specimen helped differentiate the non-neoplastic hepatic cyst from biliary cystadenoma or cystadenocarcinoma without any malignant component.

\section{Conclusions}

In conclusion, this report describes the diagnosis, treatment, and long-term follow-up of a cat with a giant hepatic cyst. The diagnosis was confirmed on the basis of radiographic, ultrasonographic, computed tomographic, and histological findings. Removal of the simple hepatic cyst together with a portion of the left lateral liver lobe resulted in a good prognosis with the regression of clinical signs and no postoperative complications.

\section{Acknowledgements}

None.

\section{Conflicts of Interest}

The authors have no conflicting interests.

\section{References}

1. Ito T, Noguchi A, Saito T, Nakashima S, Ikoma D, Shimizu T, et al. A case of biliary cystadenocarcinoma followed up as a simple cyst of the liver. Jpn J Gastroenterol Surg 2009; 42: 651-656.

2. Mazza OM, Fernandez DL, Pekolj J, Pfaffen G, Sanchez Clariá R, Molmenti EP, et al. Management of nonparasitic hepatic cysts. J Am Coll Surg 2009; 209: 733-739.

3. Miyamoto M, Oka M, Izumiya T, Nagaoka T, Ishihara Y, Ueda K, et al. Nonparasitic solitary giant hepatic cyst causing obstructive jaundice was successfully treated with monoethanolamine oleate. Intern Med 2006; 45: 621-625.

4. Nyland TG, Koblik PD, Tellyer SE. Ultrasonographic evaluation of biliary cystadenomas in cats. Vet Radiol Ultrasound 1999; 40: 300-306.

5. Ozbalci GS, Taurikulu Y, Erel S, Kismet K, Akkus MA. Giant simple hepatic cyst (a case report) and review of literature. Eur J Surg Sci 2010; 1: 53-57.

6. Proverbio D, Spada E, Faverzani S, Grieco V, Perego R, Addis A. Multiple hepatic vascular cysts in a young ragdoll cat. Vet Rec 2008; 163: 748-749.

7. Saini S, Mueller PR, Ferrucci JT Jr, Simeone JF, Wittenberg J, Butch RJ. Percutaneous aspiration of hepatic cysts does not provide definitive therapy. AJR Am J Roentgenol 1983; 141: 559-560.

8. Salemis NS, Georgoulis E, Gourgiotis S, Tsohataridis E. Spontaneous rupture of a giant non parasitic hepatic cyst presenting as an acute surgical abdomen. Ann Hepatol 2007; 6: 190-193.

9. Sato T, Imai M, Hayashi K, Isokawa O, Nomura T, Tsuchiya Y, et al. Giant hepatic cyst with septal structure: diagnosis and management. Int J Hepatol 2013; 2013: 981975.

10. Vachha B, Sun MR, Siewert B, Eisenberg RL. Cystic lesions of the liver. AJR Am J Roentgenol 2011; 196: W355-W366.

11. Washizu M, Kobayashi K, Misaka K, Hayashi T, Kinoshita G, Kondo M, et al. Surgery of hepatic cysts in a cat. J Vet Med Sci 1992; 54: 1051-1053. 